\title{
Le pronom personnel sujet dans le parler de Chantemerle-les-Blés (Drôme) Variation aléatoire ou systématique dans une zone de transition linguistique?
}

\author{
Fréchet Claudine, \\ Institut Pierre Gardette, Université Catholique de Lyon
}

\begin{abstract}
Chantemerle-les-Blés se trouve dans la partie septentrionale de la Drôme où, des parlers occitans nord provençaux, on passe aux parlers francoprovençaux. Le changement ne s'effectue pas en une seule étape, mais par des différences successives et multiples. Il ne s'agit pas d'une frontière où le parler est occitan en deçà et francoprovençal au-delà, mais bien d'une «zone frontalière » comme l'écrit JeanClaude Bouvier ${ }^{1}$.
\end{abstract}

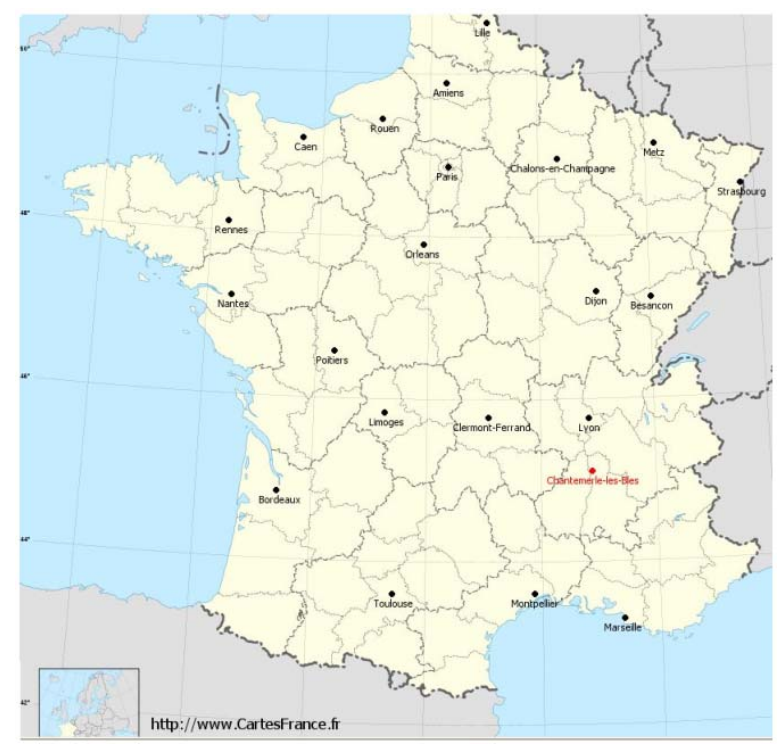

L'étude du pronom personnel sujet à partir de l'œuvre en prose d'Eloi Abert (1849-1914)², écrite dans le parler de Chantemerle-les-Blés ${ }^{3}$, peut apporter une contribution intéressante aux diverses études déjà effectuées sur la forme et l'emploi de ce pronom ${ }^{4}$. Historiquement, les pronoms personnels sont des outils qui ont été introduits, par nécessité, pour pallier l'uniformisation des désinences verbales suite aux évolutions phonétiques qui se sont succédés au cours des siècles (ex. : $1^{\text {ère }}, 2^{\mathrm{e}}, 3^{\mathrm{e}}$ personne du verbe chanter à l'indicatif présent [Jãt]). Je, tu, il... sont appelés des pronoms personnels conjoints car ils ont comme seule valeur de préciser la personne du verbe. Parallèlement, les pronoms personnels disjoints, eux, renforcent la personne (ex. : moi, toi, lui...).

A propos de l'occitan, Jean-Claude Bouvier écrit, dans l'introduction de son article « Le pronom personnel sujet et la frontière linguistique entre provençal et francoprovençal $»^{5}$, que «les différents dialectes de langue d'oc connaissent un jeu précis des désinences verbales permettant d'assurer à elles seules la distinction des personnes ». Lucien Barou, dans sa thèse sur les parlers du Forez, souligne 
également que «le paysage dialectal contemporain, oppose une syntaxe nord-occitane marquée par l'omission presque absolue du sujet pronominal à une syntaxe francoprovençale qui connaît l'expression du pronom sujet $»^{6}$. Or, à Chantemerle-les-Blés, village qui se situe en domaine occitan si l'on se réfère à la limite tracée, à partir de la non-palatalisation du $\mathrm{A}$ du latin après une consonne palatale ou devenue palatale (ex. : MANDUCARE > migear), par Gaston Tuaillon ${ }^{7}$, les pronoms personnels conjoints et disjoints sont utilisés.

Après une présentation des formes relevées dans le corpus retenu, des pistes d'explication seront proposées et comparées à celles données par Jean-Claude Bouvier.

L'emploi irrégulier du pronom personnel sujet est probablement le trait le plus explicite pour mettre en évidence la situation de Chantemerle-les-Blés dans une zone de transition. Cette irrégularité estelle systématique ou est-elle le fait de la versatilité d'Eloi Abert ? Cette dernière hypothèse ne semble pas devoir être retenue. En effet, Eloi Abert, qui fut professeur d'allemand, a lui-même établi un glossaire et une grammaire du parler de Chantemerle-les-Blés.

\section{$11^{\text {ère }}$ personne du singulier :}

Le pronom personnel conjoint de la première personne n'est pas exprimé dans les phrases de type affirmatif (ex. : eyou, jiterou, n'en savou ren, seré, veniou...), sauf lorsqu'il précède immédiatement le verbe avoir (auxiliaire ou non) à l'indicatif présent (ex. : j'ai, j'ai brougea, j'ai tant marcha, j'ai pas lési, «j'ai, j'ai réfléchi, j'ai beaucoup marché, je n'ai pas loisir »). Lorsqu'une négation ou un pronom est placé juste avant le verbe avoir, le pronom conjoint est absent (ex. : n'ai vegu, l'ai jamais rimarqua...). Dans les phrases de type interrogatif, le pronom personnel n'est pas exprimé (ex. : siou, diré, vonte siou...« je suis, je dirai, où suis-je...»). S'il l'est, c'est sous la forme -é, précédée de la consonne euphonique $-t-($ ex. : siou-t-é, rêvou-t-é, prendré-t-é...). On lit aussi la séquence : $j$ 'ai-t-é pougu ? Enfin, le pronom personnel conjoint n'est pas exprimé dans les incises.

Tableau 1

\begin{tabular}{|l|l|l|l|l|l|}
\hline \multicolumn{2}{|l|}{ Phrase affirmative } & \multicolumn{2}{l|}{ Phrase interrogative } \\
\hline & & Renforcement & Incise & & \\
\hline$J^{\prime}+a i$ & Absent & $J^{\prime}$ ai-t-é pougu & Absent & Absent & $-t-e ́$ \\
\hline
\end{tabular}

L'absence du pronom conjoint devant les verbes autres que avoir connaît une seule exception : j'ovou (dans Lou Faust Païsan), alors que nous lisons ovou ailleurs.

Le pronom personnel disjoint est mi (ex. : mi pensou pas...).

\section{$2^{\mathrm{e}}$ personne du singulier :}

Le pronom personnel conjoint de la deuxième personne est généralement exprimé (ex. : $t i$ meriré, ti me douneré, t'aurà, ti sia, ti vole...) pour les verbes transitifs et intransitifs dans les phrases de type affirmatif ou interrogatif. En revanche, il est absent de la conjugaison des verbes pronominaux (ex. : te reveillerè, te sia mouqua...). 
Tableau 2

\begin{tabular}{|l|l|l|}
\hline \multicolumn{2}{|l|}{ Phrase affirmative } & Phrase interrogative \\
\hline v. tr. ou intr. & v. pr. & \\
\hline$T i+$ consonne & Absent & $-t u$ \\
$T^{\prime}+$ voyelle & Absent & \\
\hline
\end{tabular}

Le pronom personnel conjoint de la deuxième personne est polymorphe $: t i, t^{\prime}, t u$.

Le pronom personnel disjoint n’apparaît pas dans le manuscrit utilisé pour établir le corpus.

$\underline{3^{\mathrm{e}} \text { personne du singulier : }}$

Le pronom personnel conjoint masculin de la $3^{\mathrm{e}}$ personne est celui qui offre la plus grande diversité de formes. Il est le plus souvent exprimé. Toutefois, il est absent quatre fois (ex. : aguê po, criê, nen vo fare ino této, se pendulê « il eut peur, il cria, il va en faire une tête, il se suspendit»), sans qu'il soit possible de donner une explication. En ce qui concerne aguê po, le sens est clair. En revanche, ce n'est pas le cas pour criê puisque la forme est la même au passé simple ( $3^{\mathrm{e}}$ pers. sg) et à l'indicatif présent $\left(2^{\mathrm{e}}\right.$ pers. pl.). Le pronom personnel conjoint est employé sous la forme o (ex. : o conta, o saupê...), ou (ex. : ou lous garavo « il les enlevait»), ol (ex. : ol eyô, ol anê...) et $i$ - rare - (ex. : $i$ saurê bien). En incise, il est présent sous la forme -è (ex.: dissi-t-é, feguê-t-é), comme dans les phrases de type interrogatif (ex. : vo-t-é).

Tableau 3

\begin{tabular}{|l|l|l|l|}
\hline \multicolumn{2}{|l|}{ Phrase affirmative } & Phrase interrogative \\
\hline $\begin{array}{l}\mathrm{Ol}+\text { consonne } \\
\mathrm{O}+\text { voyelle } \\
\mathrm{Ou}+\text { consonne } \\
\mathrm{I}+\text { consonne }\end{array}$ & Absent & $-t-\dot{e}$ & \\
\hline
\end{tabular}

Remarque : $o$ peut être aussi utilisé pour la politesse : o seri « vous seriez ».

Le pronom disjoint est iellou (ex. : iellou a près so man « lui, il a pris sa main »).

Pour le féminin, le pronom est systématiquement utilisé. Il apparaît sous deux formes (il ou $i$ ) dans les phrases de type affirmatif (ex. : il eyô, il o belio poussa, il é creva, i pousserê, $i$ vé « elle avait, elle a peutêtre poussé, elle est morte, elle poussera, elle voit »). I est utilisé en incise (ex. : ajoutê-t-i). En tournure interrogative, le pronom est absent (ex. : qu'épero), mais très peu de contextes se prêtent à cette observation. 
Tableau 4

\begin{tabular}{|l|l|l|}
\hline \multicolumn{2}{|l|}{ Phrase affirmative } & $\begin{array}{l}\text { Phrase } \\
\text { interrogative }\end{array}$ \\
\hline & Incise & \\
\hline $\begin{array}{l}I l+\text { consonne } \\
I+\text { voyelle }\end{array}$ & $-t-i$ & Absent \\
\hline
\end{tabular}

Le pronom disjoint est iello (ex. : iello ero deveniô).

Le pronom conjoint impersonnel qui a, comme équivalent en français «il», est souvent absent (ex. : feguê convenu, m'o sembla «il fut convenu, il m'a semblé »). Lorsqu'il est exprimé, il est présent la forme al (ex. : al era).

Tableau 5

\begin{tabular}{|l|}
\hline Phrase affirmative \\
\hline$A l+$ voyelle \\
\hline
\end{tabular}

Dans des constructions telles que es la not, es ben fini, es convenut..., le pronom n'est pas exprimé alors que, en français, on aura le démonstratif « ce ».

\section{$1^{\text {ère }}$ personne du pluriel :}

Le pronom conjoint de la première personne du pluriel est le plus souvent absent (ex. : an $a$ discutar, devan, sian pas tii, nous endeurmian, nous metteran...). Lorsqu'il est présent, ce qui est rare, il l'est sous la forme nous dans les phrases de type affirmatif (ex.: nous nous amuseran) ou, plus fréquemment sous la forme. nous autris (ex.: nous autris sautan, nous autris soulevan...). Il ya également -é en incise (ex. : vivan-t-é...) ou dans les phrases de type interrogatif (ex. : sian-t-é).

Tableau 6

\begin{tabular}{|l|l|l|l|}
\hline \multicolumn{3}{|c|}{ Phrase affirmative } & Phrase interrogative \\
\hline & & Incise & \\
\hline Nous, nous autris & Absent & $-t-e ́$ & $-t-\dot{e}$ \\
\hline
\end{tabular}

Dans notre corpus, nous n'avons pas trouvé d'attestation de la forme du pronom disjoint.

$\underline{2^{\mathrm{e}} \text { personne du pluriel : }}$

Le pronom conjoint de la deuxième personne du pluriel vous ou vous autris est toujours présent que ce soit dans des phrases de type affirmatif ou interrogatif (ex. : vous savez, vous vous leveri, vous anê, sia-vous, voulez-vous, veyê-vous...). De plus, la forme -é est parfois utilisée dans les tournures interrogatives (ex. : vous sia-t-é). 
Tableau 7

\begin{tabular}{|l|l|}
\hline Phrase affirmative & Phrase interrogative \\
\hline Vous, vous autris & Vous \\
& $-t-\grave{e}$ \\
\hline
\end{tabular}

Nous n'avons pas trouvé d'attestation de la forme du pronom disjoint dans notre corpus.

$\underline{3}$ personne du pluriel :

Le pronom conjoint de la troisième personne du pluriel (masculin ou féminin) est le plus souvent présent, sous la forme $i l$, s'il est suivi d'une voyelle (ex. : il appelan, il attacheran...) ou, sous la forme $i$, lorsqu'il est suivi d'une consonne (ex. : $i$ decideran, $i$ se mettian...). En incise, c'est la forme $-i$ qui est utilisée (ex. : disian-t-i...). Il y a quelques cas d'absence du pronom (ex. : disseran, feran...) mais ils sont rares. Cependant, si le pronom est absent, le verbe, le plus souvent, a un sujet indéfini; sa forme en incise, sera alors $-t$-é.

Tableau 8

\begin{tabular}{|l|l|l|l|}
\hline Phrase affirmative & & & Phrase interrogative \\
\hline$I l+$ voyelle & Incise & $-t-i$ & $-t-i$ (ils, elles) \\
$I+$ consonne & Absent & Absent & $-t-e ́($ on) \\
\hline
\end{tabular}

Les formes de pronoms disjoints présentes sont ielli pour le masculin. Le corpus ne comporte pas de pronom disjoint de la troisième personne du pluriel pour le féminin.

A partir de ces éléments, il est possible de dresser un tableau récapitulatif qui facilitera le repérage des particularités de l'emploi du pronom personnel sujet dans le parler de Chantemerle-les-Blés. 
Tableau 9

\begin{tabular}{|c|c|c|c|c|c|c|c|}
\hline & \multicolumn{6}{|c|}{ Formes conjonctives } & \multirow{2}{*}{\begin{tabular}{|l} 
Formes \\
disjonctives
\end{tabular}} \\
\hline & Présence & Absence & $\begin{array}{l}\text { Phrase } \\
\text { affirmative }\end{array}$ & $\begin{array}{l}\text { Phrase } \\
\text { interrogative }\end{array}$ & Incise & Formes & \\
\hline $\begin{array}{l}1^{\text {ère }} \\
\text { pers. } \\
\text { sg }\end{array}$ & $\begin{array}{l}\text { j'ai } \\
\text { (une } \\
\text { exception) }\end{array}$ & $\begin{array}{l}\text { Constante } \\
\text { (saufj'ai) }\end{array}$ & $J^{\prime}, \varnothing$ & $\varnothing,-\mathrm{t}-\dot{e}$ & $\varnothing$ & $\varnothing, j^{\prime},-\mathrm{t}-\dot{e}$, & $m i$ \\
\hline $\begin{array}{l}2^{\mathrm{e}} \text { pers. } \\
\text { sg. }\end{array}$ & $\begin{array}{l}\text { V. transitifs } \\
\text { et } \\
\text { intransitifs }\end{array}$ & v. pr. & $\begin{array}{l}t i \\
\text { consonne } \\
t^{\prime}+\text { voyelle }\end{array}$ & $-t u$ & Pas d'exemple & $\varnothing, t i, t^{\prime}, t u$ & Pas d'exemple \\
\hline $\begin{array}{l}3^{\mathrm{e}} \text { pers. } \\
\text { m. sg. }\end{array}$ & $\begin{array}{l}\text { Le plus } \\
\text { souvent }\end{array}$ & $\begin{array}{l}\text { Quelques } \\
\text { fois }\end{array}$ & $\begin{array}{l}\varnothing, \\
O l+\text { voyelle } \\
O \\
\text { consonne } \\
O u+ \\
I+ \\
\end{array}$ & $-\mathrm{t}-\dot{e}$ & -t-é & $\begin{array}{l}\varnothing, o l, o, o u, i, \\
\text {-t-é }\end{array}$ & iellou \\
\hline $\begin{array}{l}3^{\text {e }} \text { pers. } \\
\text { f. sg. }\end{array}$ & $\begin{array}{l}\text { Constante } \\
\text { (une } \\
\text { exception) }\end{array}$ & Jamais & $\begin{array}{l}I l+\text { voyelle } \\
I+\text { consonne }\end{array}$ & $-\mathrm{t}-i$ & Pas d'exemple & $I l, i$ & Iello \\
\hline $\begin{array}{l}3^{\mathrm{e}} \text { pers. } \\
\text { imp. }\end{array}$ & Souvent & Souvent & $\begin{array}{l}\varnothing, \\
A l+\text { voyelle }\end{array}$ & $\begin{array}{l}\text { Pas } \\
\text { d'exemple }\end{array}$ & $\begin{array}{ll}\mathrm{Ne} & \text { s'applique } \\
\text { pas }\end{array}$ & $\varnothing, a l$ & $\begin{array}{ll}\mathrm{Ne} & \text { s'applique } \\
\text { pas } & \end{array}$ \\
\hline $\begin{array}{l}1^{\text {ère }} \\
\text { pers. } \\
\text { pl. }\end{array}$ & Rarement & $\begin{array}{l}\text { Le plus } \\
\text { souvent }\end{array}$ & $\begin{array}{ll}\varnothing, \\
\text { Nous, nous } \\
\text { autris }\end{array}$ & $-\mathrm{t}-\dot{e}$ & -t-é & $\varnothing$, nous, -t-é & Pas d'exemple \\
\hline $\begin{array}{l}2^{\mathrm{e}} \text { pers. } \\
\text { pl. }\end{array}$ & Constante & Jamais & \begin{tabular}{|ll} 
Vous, vous \\
autris
\end{tabular} & Vous, -t-é & $-\mathrm{t}-\dot{e}$ & Vous, -t-é & Pas d'exemple \\
\hline $\begin{array}{l}3^{\mathrm{e}} \text { pers. } \\
\text { m. pl. }\end{array}$ & $\begin{array}{l}\text { Le plus } \\
\text { souvent }\end{array}$ & Rarement & $\begin{array}{l}\varnothing, \\
I l+\text { voyelle } \\
I+\text { consonne }\end{array}$ & $-\mathrm{t}-i$ & $-\mathrm{t}-i$ & $\varnothing, i l,-\mathrm{t}-i$ & $\begin{array}{l}\text { Absent } \\
\text { corpus }\end{array}$ \\
\hline $\begin{array}{l}3^{\mathrm{e}} \text { pers. } \\
\text { f. pl. }\end{array}$ & Constante & Jamais & $\begin{array}{l}I l+\text { voyelle } \\
I+\text { consonne }\end{array}$ & $-\mathrm{t}-i$ & $-\mathrm{t}-i$ & $I l,-\mathrm{t}-i$ & $\begin{array}{l}\text { Absent } \\
\text { corpus }\end{array} \quad \mathrm{du}$ \\
\hline $\begin{array}{l}3^{\mathrm{e}} \text { pers. } \\
\text { pl. } \\
\text { «on » }\end{array}$ & Jamais & Constante & $\varnothing$ & $-\mathrm{t}-i$ & -t-é & $\varnothing,-\mathrm{t}-i$ & $\begin{array}{ll}\mathrm{Ne} & \text { s'applique } \\
\text { pas } & \end{array}$ \\
\hline
\end{tabular}


Remarque : Les exceptions répertoriées ne prêtent pas à confusion. La désinence verbale permet de situer la forme dans la conjugaison (ex. : ai acheta, qu'éperero).

Il apparaît que le parler de Chantemerle-les-Blés porte les marques de diverses influences. Pour plusieurs personnes, l'emploi ou le non-emploi est systématique. Toutefois, la variation du pronom est présente selon la forme ou la nature du verbe conjugué (initiale vocalique ou consonantique du verbe, verbe transitif ou pronominal).

Il y a donc des éléments du système linguistique qui sont fixes et non aléatoires. Tout d'abord, selon les éléments du corpus, les formes disjointes semblent distinctes des formes conjointes - nous n'avons cependant pas d'élément pour la $2^{\mathrm{e}}$ personne du singulier -. Ensuite, comme dans les parlers occitans, le pronom conjoint est absent. C'est le cas de la

$1^{\text {ère }}$ pers., phrase affirmative (excepté pour le v. avoir à l'ind. prés.)

$2^{\mathrm{e}}$ pers., phrase affirmative (pour les v. pron.)

$3^{\mathrm{e}}$ pers. pl. ind., phrase affirmative.

Mais, comme dans les parlers francoprovençaux ou d'oïl, le pronom conjoint est présent pour la

- $1^{\text {ère }}$ pers. du sing. du verbe avoir à l'indicatif présent. Le pronom assure probablement au verbe (ai [e]) plus de volume sonore. En effet, ce pronom est systématiquement présent lorsque le verbe est au présent de l'indicatif. Il est absent pour les autres temps où la forme verbale est toujours constituée de plusieurs phonèmes (ex. : aguê po, aguérou, aguesse). En ce qui concerne les autres verbes, la désinence verbale suffit à indiquer la personne.

$-2^{\mathrm{e}}$ pers. du sing. Le pronom conjoint n'est présent que lorsque la forme verbale ne comporte pas dans sa désinence l'indication évidente de la personne. Pour les verbes pronominaux, le pronom objet est exprimé et donne les indications utiles ; le pronom conjoint serait alors redondant.

- $3^{\mathrm{e}}$ pers. m. sg.. Il est difficile de justifier l'emploi ou non de ce pronom. Il est évident qu'il est très fréquent et que les formes multiples des désinences ne permettent pas toujours de repérer la personne. Pour la $3^{\mathrm{e}}$ pers., de façon générale, que ce soit au masculin ou au féminin, au singulier ou au pluriel, le pronom est presque toujours exprimé, au masculin, ou toujours exprimé, au féminin. L'emploi ou non du pronom conjoint est déterminant pour la compréhension, à quelques exceptions près. En effet, lorsque le pronom conjoint est exprimé, il s'agit toujours du masculin ou du féminin, alors que le pronom conjoint est toujours absent lorsque le verbe est à la forme impersonnelle («il »).

- $1^{\text {ère }}$ pers. du pl. Même si c'est de façon aléatoire, que ce soit pour des verbes transitifs, intransitifs ou pronominaux, son expression est justifiée par le fait que la désinence est identique à celle de la $3^{\mathrm{e}}$ pers. du pluriel.

$-2^{\mathrm{e}}$ pers. du pl. Son expression est systématique. Il est possible que cela soit dû au fait que que les désinences verbales sont multiples (ex. : sia, seri, -ê) et que le risque de confusion serait élevé en l'absence du pronom conjoint.

Le pronom conjoint est donc présent là où il s'agit de lever une ambiguïté. Il ne s'agit pas de surcharger la syntaxe de la phrase mais bien d'apporter une précision grammaticale.

La présence ou l'absence du pronom conjoint dans le parler de Chantemerle-les-Blés répond à des exigences. Cependant, outre l'emploi, la forme des pronoms doit être aussi examinée car elle est variable. Mais, là encore, y a-t-il une variation raisonnée ou une variation aléatoire ? 
Les formes du pronom conjoint diffèrent souvent en fonction du type de la phrase. En tournure interrogative, une même forme est présente à plusieurs personnes : -t-é. Il s'agit, probablement, d'une généralisation de la consonne $-\mathrm{t}-$ que l'on retrouve par exemple dans la construction française « aime-til » qui est une trace de la terminaison latine de la $3^{\mathrm{e}}$ pers. du singulier (AMAT). Té est peut-être une sorte de pronom «passe-partout», selon la terminologie de Monseigneur Gardette ${ }^{8}$. Té est néanmoins absent pour la $3^{\mathrm{e}}$ pers. du pl. et coexiste, pour les autres personnes, avec des pronoms spécifiques. Le parler de Chantemerle-les-Blés ne peut donc pas illustrer l'affirmation de Lucien Barou selon laquelle nous avons des « pronoms spécifiques au nord de l'isoglosse [...] pronom passe-partout au sud $»^{9}$.

Seules les $3^{\mathrm{e}}$ pers. féminin singulier, masculin et féminin pluriel et la $2^{\mathrm{e}}$ personne du pluriel ont le même pronom dans la phrase affirmative et dans la phrase interrogative, même si, pour la $2^{\mathrm{e}}$ personne, on trouve aussi exceptionnellement la forme $-t-e ́$.

La $2^{\mathrm{e}}$ personne du singulier se distingue des autres par la répartition des formes du pronom conjoint. Ti ou $t$ ' sont utilisés dans les phrases de type affirmatif, $t u$ est utilisé dans les phrases de type interrogatif. Le pronom $t u$ est sans doute dû à une influence du français.

La $3^{\text {e }}$ personne du singulier est aussi particulière par ses multiples formes : ol, o, ou, $i . O l$ et $o$ sont les formes les plus courantes. $O u$ est attesté deux fois devant consonne. $I$, pour le masculin, semble être dû à l'influence du français et c'est sans doute un emploi fautif de l'auteur plus qu'une forme du parler. Le texte semble orienter l'analyse dans ce sens : son petit que compreniô pas que so mêre se trouvavo ma [...] i se mettê à plourar et i se sarravo contro mas chambas coume s'i cherchavo de secôr. In quart d'huro après, il ero reveniô à iello. Si l'on suit la grammaire proposée par Eloi Abert, il convient de traduire ainsi : « son petit qui ne comprenait pas que sa mère se trouvait mal [...] elle se mit à pleurer et elle se serrait contre mes jambes comme si elle cherchait du secours. Un quart d'heure après, elle était revenue à elle ». I est alors traduit par "elle". Or, le contexte montre que la mère s'est évanouie et que c'est le petit garçon de quatre ou cinq ans qui se serre contre les jambes de la narratrice. Il convient donc de retenir, comme formes possibles du pronom personnel conjoint de la $3^{\mathrm{e}}$ personne masculin singulier : ol, $o$ ou ou.

Une fois encore, il y a variation dans les formes mais il y a régularité dans l'emploi de ces formes. «Le système du pronom personnel sujet, que les parlers soient dits nord-provençaux ou francoprovençaux, est un système à deux éléments (ou deux séries) : il distingue toujours formes prédicatives (ou disjointes) et formes non-prédicatives (ou conjointes) $»^{10}$ «[...] du point de vue syntaxique c'est évident, on l'a vu : l'existence de la série double entrâne automatiquement en langue l'emploi obligatoire de la forme conjointe du verbe [...] Dans le discours ou dans les variétés régionales de la langue c'est plus complexe $»^{11}$.

Si l'on met en regard les éléments relevés chez Eloi Abert et ceux qui l'ont été par Jean-Claude Bouvier dans le parler de Chantemerle-les-Blés, on peut noter une influence du français chez Eloi Abert. JeanClaude Bouvier cite lv saya té «le savais-tu», alors qu'Eloi Abert utilise le pronom conjoint $t u$ dans un énoncé de type interrogatif. De plus, la construction des phrases interrogatives d'Eloi Abert, à une ou deux exceptions près, ne correspond pas au schéma occitan où le verbe est situé en fin de phrase et où le pronom conjoint est absent.

Quant à la distinction entre la $1^{\text {ère }}$ personne du pluriel et la $3^{\mathrm{e}}$ personne du pluriel, elle est marquée par une désinence différente dans plusieurs parlers de la Drôme (ex. : -an première personne, -on troisième personne). Dans le parler de Chantemerle-les-Blés, comme dans un certain nombre de parlers nordprovençaux, la désinence est la même pour ces deux personnes et la forme verbale est accentuée sur le radical. Le pronom, lorsqu'il est exprimé, est donc bien «la seule marque distinctive de la personne $»^{12}$ à moins que le sujet ne soit pas pronominalisé. Il y a donc bien ici, pour reprendre la formulation de Lucien Barou, « une corrélation entre l'emploi des formes spécifiques du pronom sujet et le déclin de la déclinaison verbale $»^{13}$. Si l'on suit Paul Meyer ${ }^{14}$ et Robert Lafont ${ }^{15}$, cela peut amener à considérer cette 
uniformisation des désinences comme un archaïsme. Jean-Claude Bouvier propose « une poussée des parlers septentrionaux [...] qui précisément ont conservé - an au présent ${ }^{16}$.

Néanmoins, à la lecture des textes d'Eloi Abert, il n'est pas possible de reprendre à notre compte l'affirmation de Jean-Claude Bouvier, selon laquelle Chantemerle-les-Blés se situerait dans «un ensemble de parlers qui possède une structure morphologique et syntaxique qui l'apparente au francoprovençal et à la langue d'oïl et l'éloigne du système linguistique de la langue d'oc $»^{17}$. En effet, l'emploi de la forme conjointe n'est pas systématique. Nous l'avons déjà montré, nous sommes réellement dans un système intermédiaire où la structure occitane peut être présente sur le plan phonétique, tout comme l'absence du pronom personnel sujet conjoint. En effet, sur les dix cas présentés, sept contextes d'absence de pronom sont répertoriés. Eloi Abert n'utilise de façon systématique, et dans tous les contextes, que le pronom conjoint vous. Néanmoins, ce pronom peut apparaître aussi sous la forme -t-é dans la phrase interrogative. Le pronom personnel de la troisième personne n'est pas toujours employé. Selon Jean-Claude Bouvier, «Dans les parlers drômois qui possèdent le système francoprovençal du pronom personnel, la distinction entre les deux < pronom personnel et pronom impersonnel $>$ est constante. Parfois, c'est l'absence pure et simple de pronom, c'est-à-dire un pronomzéro, qui caractérisera l'impersonnel [...] Mais le plus souvent c'est la forme $a l$ ou $a^{18}[\ldots]$ qui sert de support aux verbes unipersonnels [...] la diversité des réalisations géographiques serait là aussi à examiner ${ }^{19}$ ». Or, le parler utilisé par Eloi Abert ne présente pas de «constante » dans l'emploi des pronoms personnels sujets conjoints. Le pronom personnel et impersonnel de la $3^{\mathrm{e}}$ personne peut être présent ou absent. Les seules constantes, pour cette personne, sont la présence du pronom personnel conjoint au féminin et son absence, lorsqu'il peut être traduit en français par « il » (impersonnel).

Le parler utilisé par Eloi Abert et le parler présenté par Jean-Claude Bouvier sont séparés par cinq générations d'utilisateurs qui ont fait évoluer la langue.

Tableau 10

Utilisation et formes de l'emploi personnel sujet conjoint à Chantemerle-les-Blés aux environs de 1870 et aux environs de 1971

\begin{tabular}{|c|c|c|}
\hline & $\begin{array}{l}\text { Formes présentes dans les textes d'Eloi } \\
\text { Abert }\end{array}$ & $\begin{array}{l}\text { Formes relevées auprès de locuteurs } \\
\text { par Jean-Claude Bouvier }\end{array}$ \\
\hline $1^{\text {ère }}$ pers. sg & $\varnothing$, j', -t-é, & $\varnothing, \mathrm{j}^{\prime}, \mathrm{a}$ \\
\hline $2^{\mathrm{e}}$ pers. sg. & $\varnothing, \mathrm{ti}, \mathrm{t}$ ', tu (interr.) & $\mathrm{t} \mathrm{i} / \mathrm{te}+$ consonne, $\mathrm{t}$ ', té (interr.) \\
\hline $3^{\mathrm{e}}$ pers. m. sg. & $\varnothing$, ol, o, ou, i, -t-é & ó, ól \\
\hline $3^{\mathrm{e}}$ pers. f. sg. & $\mathrm{Il}, \mathrm{i}$ & $\mathrm{i}, \mathrm{il}$ \\
\hline $3^{\mathrm{e}}$ pers. imp. & $\varnothing$, al & a, al \\
\hline $1^{\text {ère }}$ pers. pl. & $\varnothing$, nous, -t-é & nous, j', a \\
\hline $2^{\mathrm{e}}$ pers. pl. & Vous, -t-é & vous, \\
\hline $3^{\mathrm{e}}$ pers. m. pl. & $\varnothing$, il, -t-i & I, il \\
\hline $3^{\mathrm{e}}$ pers. f. pl. & Il, -t-i & $\mathrm{I}, \mathrm{il}$ \\
\hline
\end{tabular}


En un siècle, l'emploi systématique du pronom conjoint s'est généralisé si ce n'est à la première personne du singulier. Le pronom $j$ ' s'est étendu à la première personne du pluriel. Le pronom $a$ peut être utilisé pour trois personnes sans pour autant créer d'ambiguité, puisque les désinences verbales de la première et de la troisième personne au singulier, ainsi que de la première personne au pluriel, sont distinctes. Ce pronom n'a pas de valeur de trait distinctif, il s'est sans doute étendu pour assurer la régulation du système de la séquence : pronom sujet conjoint + verbe. Cet emploi pourrait bien être la preuve que l'absence du pronom a été ressentie comme un manque.

\section{$* * * * *$}

Cette étude corrobore ce qu'affirme Jean-Claude Bouvier : « la frontière linguistique traditionnelle entre langue d'oc et langue d'oïl ou francoprovençal et la limite d'emploi des formes conjointes du pronom personnel sujet ne coïncident que rarement $»^{20}$.

Dans le parler de Chantemerle-les-Blés, le pronom conjoint peut être absent, comme dans les parlers occitans. Mais l'on peut dire aussi que le pronom conjoint est présent, et cela s'applique à toutes les personnes, comme dans les parlers francoprovençaux ou d'oïl. La langue utilisée par Eloi Abert est un occitan influencé par les parlers du Nord et du Sud. Les influences septentrionales peuvent être francoprovençales et surtout françaises. En effet, dans les parlers francoprovençaux voisins l'emploi et la forme du pronom sujet est fluctuant, comme à Chantemerle-les-Blés, et ce n'est qu'en français que l'emploi du pronom est obligatoire. Comme les parlers francoprovençaux, les parlers nord-occitans, de la vallée du Rhône, sont influencés par le français. La présence et la forme des pronoms personnels sujets conjoints chez Eloi Abert est, dans l'ensemble, plus prévisible et plus pertinente que dans la même langue décrite par Jean-Claude Bouvier cent ans plus tard. Une évolution syntaxique a eu lieu, il ne s'agit pas réellement d'une simplification mais plutôt d'une systématisation de l'emploi du pronom sujet. L'emploi de ce pronom à Chantemerle-les-Blés montre la variation par rapport à une norme et ne semble pas pouvoir être retenu comme trait distinctif pour déterminer la famille linguistique à laquelle se rattache le parler étudié.

\section{Références bibliographiques}

Barou, L. (1978). Expression et omission du pronom sujet en Forez dans les parlers voisins de la limite linguistique, Thèse de dialectologie, Université des Langues et Lettres de Grenoble, p. 48.

Bouvier, J.-Cl. (1971). « Le pronom personnel sujet et la frontière linguistique entre provençal et francoprovençal ». Revue de Linguistique Romane, vol. 35, pp. 1-16

Diémoz, F. (2009). «Le pronom personnel sujet de la 3e personne du singulier et le sujet neutre en francoprovençal valaisan : étude morphosyntaxique », in Langues et cultures de France et d'ailleurs, Lyon, P.U.L., pp. 177-193.

Faure, R., Oliviéri, M. (2013), «Stratégies de topicalisation en occitan », Corpus 12, 231-270 ;

Fréchet, Cl. (2013) (édition et présentation). Eloi Abert, Récits et contes du Dauphiné, Lyon, EMCC.

Gardette, P. (1941). Etudes de géographie morphologique sur les patois du Forez, Mâcon, Protat, p. 30.

Hinzelin, M.-O., Kaiser, G.-A. (2007). « Le paramètre du sujet nul dans les variétés dialectales de l'occitan et du francoprovençal », communication orale lors du colloque GalRom07, diachronie du gallo-roman. Evolution de la phonologie et de la morphologie du français, du francoprovençal et de l'occitan (Nice, 15-16 janvier 2007), (http://www.hinzelin.net/resources/hinzelin kaiser 2007_article.pdf). 
Kaiser, G. A., Oliviéri M., Palasis, K. (2013), « Impersonal constructions in northern Occitan », in X. Álvarez Pérez, E. Carrilho, C. Magro (éds) (2013), Current Approaches to Limits and Areas in Dialectology, Cambridge, Cambridge Scholars Press, pp. 345-366.

Kristol, A. (2009). «La morphosyntaxe du pronom personnel sujet de la première personne du singulier en francoprovençal valaisan : comment manier le polymorphisme d'une langue dialectale ? », in Langues et cultures de France et d'ailleurs, Lyon, PUL, pp.195-216.

Lafont, R. (1967). La Phrase occitane, Paris, P.U.F., 1967, p. 139.

Martin, J.-B. (1974). «Le pronom personnel de la 3e personne en francoprovençal central (formes et structures) », in Travaux de Linguistique et de Littérature (TraLiLi), 12/1, Paris, Klincksieck, pp. 85-116.

Meyer, P. (1880). « Les troisièmes personnes du pluriel en provençal, in Romania, Paris, Vieweg, vol. 9, pp. 192-215

Savoia, L. M., Rita Manzini (2010), «Les clitiques sujets dans les variétés occitanes et francoprovençales italiennes », Corpus, 9, 165-190.

Tuaillon, G. (1964). « Limite nord du provençal à l'Est du Rhône », in Revue de Linguistique Romane, vol. 28, pp. 127-142.

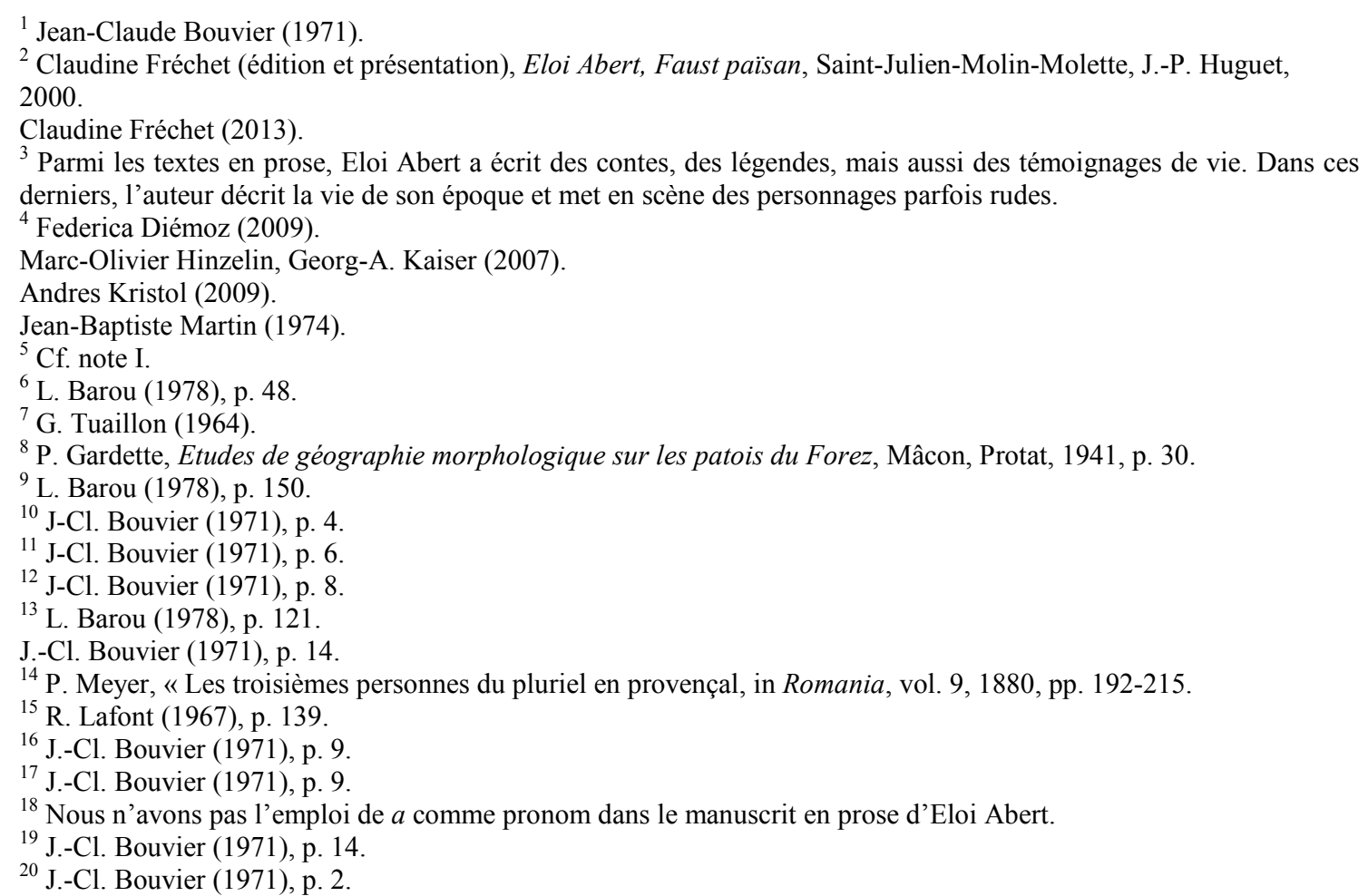

